\title{
Crystal Structure of the Bacillus subtilis Penicillin-binding Protein $4 a$, and its Complex with a Peptidoglycan Mimetic Peptide
}

\author{
Eric Sauvage $^{1 \star}$, Colette Duez ${ }^{1}$, Raphaël Herman ${ }^{1}$, Frédéric Kerff ${ }^{1}$ \\ Stephanie Petrella ${ }^{2}$, John W. Anderson ${ }^{3}$, S. A. Adediran ${ }^{3}$, R. F. Pratt ${ }^{3}$ \\ Jean-Marie Frère ${ }^{1}$ and Paulette Charlier ${ }^{1}$
}

\author{
${ }^{1}$ Centre d'Ingénierie des \\ Protéines, Université de Liège \\ Institut de Physique B5 et \\ Institut de Chimie B6a \\ Sart Tilman, B-4000 Liège \\ Belgium \\ ${ }^{2}$ Laboratoire de Recherche \\ Moléculaire sur les \\ Antibiotiques, LRMA INSERM \\ U655, Université Pierre et \\ Marie Curie, AP-HP CHU \\ Pitié-Salpêtrière, Paris, France \\ ${ }^{3}$ Department of Chemistry \\ Wesleyan University \\ Middletown, CT 06459, USA
}

\begin{abstract}
The genome of Bacillus subtilis encodes 16 penicillin-binding proteins (PBPs) involved in the synthesis and/or remodelling of the peptidoglycan during the complex life cycle of this sporulating Gram-positive rod-shaped bacterium. PBP4a (encoded by the dacC gene) is a low-molecular mass PBP clearly exhibiting in vitro DD-carboxypeptidase activity. We have solved the crystal structure of this protein alone and in complex with a peptide (D- $\alpha-$ aminopymelyl- $\varepsilon$-D-alanyl-D-alanine) that mimics the C-terminal end of the Bacillus peptidoglycan stem peptide. PBP4a is composed of three domains: the penicillin-binding domain with a fold similar to the class A $\beta$-lactamase structure and two domains inserted between the conserved motifs 1 and 2 characteristic of the penicillin-recognizing enzymes. The soaking of PBP4a in a solution of D- $\alpha$-aminopymelyl-e-D-alanyl-D-alanine resulted in an adduct between PBP4a and a D- $\alpha$-aminopimelyl- $\varepsilon$-D-alanine dipeptide and an unbound D-alanine, i.e. the products of acylation of PBP4a by D- $\alpha$-aminopymelyl-e-D-alanyl-D-alanine with the release of a D-alanine. The adduct also reveals a binding pocket specific to the diaminopimelic acid, the third residue of the peptidoglycan stem pentapeptide of B. subtilis. This pocket is specific for this class of PBPs.
\end{abstract}

(C) 2007 Elsevier Ltd. All rights reserved.

Keywords: penicillin-binding protein; DD-peptidases; Bacillus subtilis; peptidoglycan; diaminopimelic acid

\section{Introduction}

Cell wall peptidoglycan is present in most eubacteria and is required to maintain cell shape and protect the bacterium from osmotic shock. It consists of glycan chains of alternating $N$-acetylglucosamine and $N$-acetylmuramic acid cross-linked by short peptides attached to the $\mathrm{N}$-acetylmuramic acid. ${ }^{1}$ Penicillin-binding proteins (PBPs) are responsible for

Abbreviations used: $\mathrm{PBP}$, penicillin-binding protein; PBP-A, class A high molecular mass PBP; PBP-B, class B high molecular mass PBP; PBP-C, class $C$ low molecular mass PBP; R39, Actinomadura sp. strain R39 DD-peptidase; R61, Streptomyces R61 DD-transpeptidasecarboxypeptidase.

E-mail address of the corresponding author: Eric.Sauvage@ulg.ac.be the polymerization and cross-linking of the peptidoglycan. The PBPs have been divided into three classes based on sequence similarities. ${ }^{2}$ Class A high molecular mass PBPs (PBPs-A) are bifunctional enzymes with transglycosylase and transpeptidase activity. Class B high molecular mass PBPs (PBPs-B) have a C-terminal transpeptidase domain associated with one (or more) other domain(s), the function(s) of which remain(s) unknown. PBPs-B are required for cell septation and maintenance of cell shape. ${ }^{3}$ The low molecular mass PBPs (PBPs-C) form the third class of PBPs. A role in peptidoglycan maturation has been proposed for these proteins. Although generally described as monofunctional enzymes, crystallographic structures of low molecular mass PBPs have shown that they may have two (Escherichia coli $\mathrm{PBP}^{4}$ and Streptococcus pneumoniae $\mathrm{PBP}^{5}$ ) or even three domains (Actinomadura R39 DD-peptidase $^{6}$ ). The low -molecular mass PBPs can be divided into three subclasses. The PBPs of subclass 
C1 (PBPs-C1), with E. coli PBP4 and the R39 DDpeptidase (R39) as representative members, are multimodular enzymes with one or two domains inserted in the penicillin-binding domain. The PBPs of subclass C2 (PBPs-C2) are monomodular and resemble the class $C \beta$-lactamases. The DD-peptidase from Streptomyces R61 (R61), a structurally wellstudied enzyme, is a typical PBP-C2. ${ }^{7}$ The PBPs of subclass C3 (PBPs-C3) are mono or bimodular enzymes. Examples of this class are given by the $E$. coli PBP5 and the S. pneumoniae PBP3, two bimodular enzymes and by the monomodular Streptomyces K15 DD-transpeptidase. ${ }^{8}$ Globally PBPs-C act as DDcarboxypeptidase, DD-transpeptidase or DD-"endopeptidase". Up to now, it is unclear which of these activities is predominant in vivo.

Because of its elaborated life cycle, B. subtilis possesses a large number (16) of PBPs that could play a role at different stages of the $B$. subtilis development and/or be redundant in function. PBP1 (product of the gene $p o n A), \mathrm{PBP} 2 \mathrm{c}(p b p F), \mathrm{PBP} 4(p b p D)$ and PBP2d ( $p b p G)$ belong to class A. PBP2a $(p b p A)$, PBP2b $(p b p B)$, PBP3 $(p b p C)$, SpoVD (spoVD), PBPH (ykua) and PBP4b (yrrR) belong to class B. PBP4a $($ dacC $)$ is a PBP-C1, PBP4* $(p b p E)$ and PBPX ( $p b p X)$ are PBPs-C2 and PBP5 $(d a c A), \mathrm{PBP}^{*}(d a c B)$ and PBPI $(d a c F)$ are PBPs-C3. Here we describe the structure of PBP4a, the only PBP-C1 in B. subtilis.

The carboxypeptidation and transpeptidation reactions catalyzed by PBPs follow a three-step mechanism: the rapid reversible formation of a non-covalent Henri-Michaelis complex between the enzyme and a peptidoglycan stem peptide, called the donor strand, is followed by the attack of the active serine on the carbonyl carbon atom of the Cterminal D-Ala-D-Ala peptide bond, leading to the formation of an acyl-enzyme intermediate and the concomitant release of the C-terminal D-Ala (acylation). The acylation mechanism implies the abstraction of a proton from the active site serine, and this is accomplished either by the lysine residue of the SxxK motif or the carboxylate group of the substrate. $^{9-13}$ The final step (deacylation) consists of either hydrolysis, with release of the shortened peptidoglycan strand (carboxypeptidation), or cross-link formation with a second peptidoglycan stem peptide called the acceptor strand (transpeptidation). ${ }^{14}$ The residue(s) responsible for the increase of the nucleophilicity of the active serine hydroxyl group necessary for the deacylation to proceed are not clearly identified. The lysine from the SxxK motif, the serine of the SxN motif or the lysine of the KTG motif are likely candidates. ${ }^{11}$

Endopeptidation and transpeptidation are reverse activities. The DD-endopeptidase activity of the low molecular mass PBPs consists of the hydrolysis of the D-alanyl-e-meso-2,6-diaminopimelyl cross-bridge resulting from the DD-transpeptidase activity. The diaminopimelic acid ammonium-carboxylate terminal group is in a D configuration and is structurally equivalent to the leaving D-alanine. The DD-endopeptidase activity is thus a facet of the DD-carboxypeptidase activity.
To date, most of the structural analysis has been performed on the PBP-C2 R61 using a synthetic Streptomyces type peptidoglycan mimetic peptide, ${ }^{15}$ and on the E. coli PBP5 bound to a tripeptide boronic acid inhibitor. ${ }^{11}$ McDonough et al. obtained two high resolution structures with R61, one showing the noncovalent binding of the two products of the carboxypeptidase reaction and the other showing the enzyme-substrate Henri-Michaelis complex resulting from the utilization of an inactivated enzyme. However R61 might be considered as an unconventional PBP. The PBPs active site is defined by three structurally conserved motifs, SxxK, SxN and K(T/S)G but two exceptions occur in R61: the first residue of the second motif is a tyrosine and the first residue of the third motif is a histidine. It is therefore useful to obtain structural results on the DD-transpeptidase/carboxypeptidase reaction with a more conventional PBP like PBP4a. The active site architecture of E. coli PBP5 is closer to that of PBP4a than R61, and Nicola et al. reported a $1.6 \AA$ resolution structure of PBP5 in complex with a substrate-like peptide boronic acid that provides the best comprehensive model for the carboxypeptidation reaction by a low molecular mass PBP.

D- $\alpha$-Aminopymelyl- $\varepsilon$-D-alanyl-D-alanine, which mimics the C-terminal end of the $B$. subtilis peptidoglycan stem peptide, was synthesized ${ }^{16}$ and shown to interact with the PBP4a active site. We present the crystal structure of PBP4a and the result of a PBP4a crystal soaked in a solution of this peptidoglycan mimetic peptide, and discuss the implication for the carboxy/endo/transpeptidation reactions catalyzed by this enzyme.

\section{Results}

\section{PBP4a structure}

PBP4a crystallizes in space group $P 3_{2} 12$ with one molecule in the asymmetric unit. The Actinomadura R39 DD-peptidase was used as model to determine the PBP4a structure by molecular replacement (see sequence alignment on Figure 1(a)). The overall PBP4a structure is composed of three domains, one of which possesses the typical structure and active site of penicillin-binding proteins (Figure 1(b)). This domain is composed of a five-stranded antiparallel $\beta$-sheet covered on both faces by $\alpha$-helices, a structure found in all PBPs and serine $\beta$-lactamases. Between the conserved motifs 1 and 2 characteristic of this family of proteins, a large insert of 221 residues forms domains II and III, domain III being inserted into domain II. Each of the three domains possesses a $\beta$-sheet approximately oriented at a right angle to the others. The relative orientations of the three domains are similar in PBP4a, R39 and E. coli PBP4, $4^{6,17}$ (Figure 1(c)). The root mean square deviation (rmsd) between PBP4a and R39 is $1.25 \AA$ and $2.25 \AA$ between PBP4a and E. coli PBP4. 


\section{Penicillin-binding domain}

The active site is located at the interface between the five-stranded $\beta$-sheet and a cluster of $\alpha$-helices within the penicillin-binding domain (Figure 1(d)). The first conserved motif of the PBP4a active site (SSLK) begins at position 52, the second motif (SNN) is found at positions 299-301 and the third motif

(a)
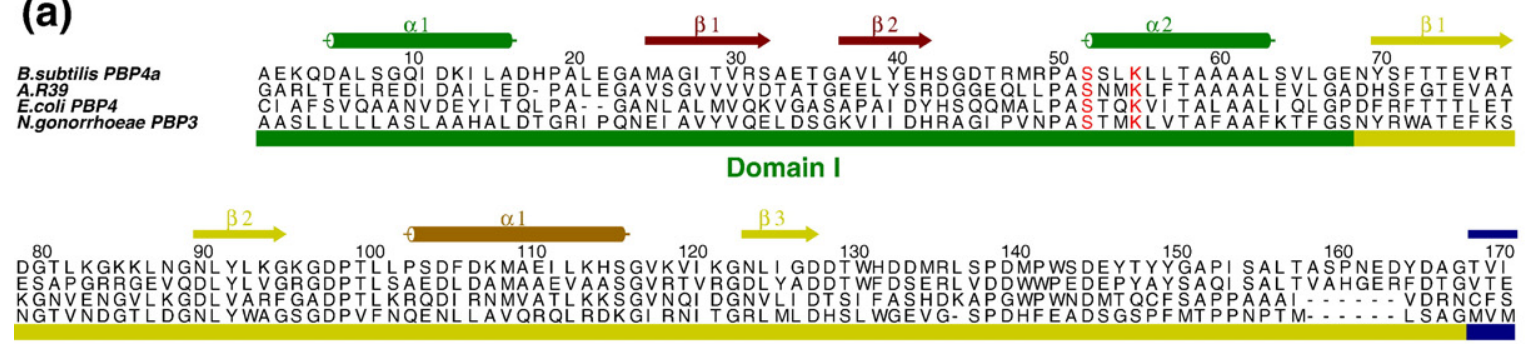

Domain II



Domain III

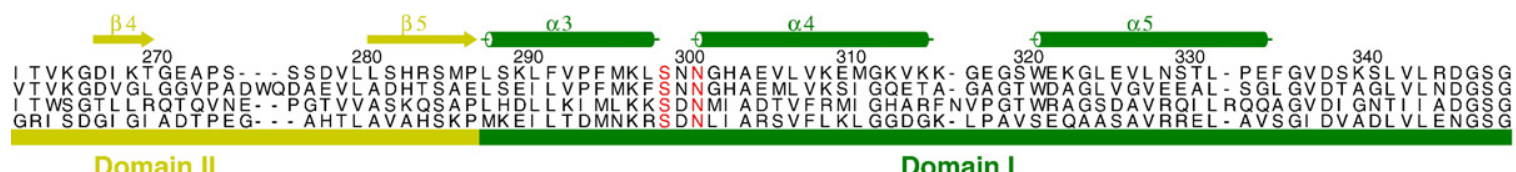

Domain II Domain I

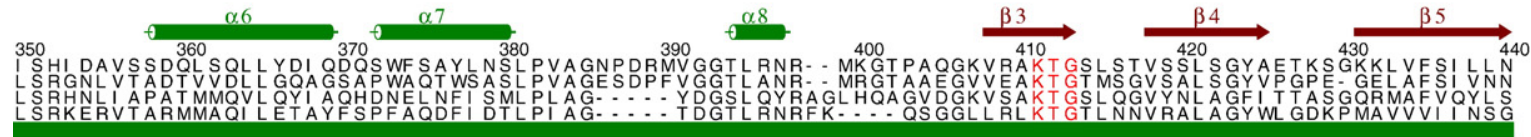

Domain I

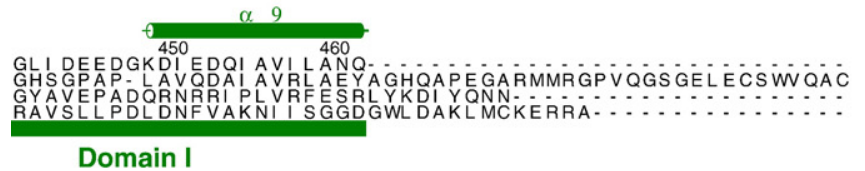

Domain I

(b)

Penicillin-binding domain

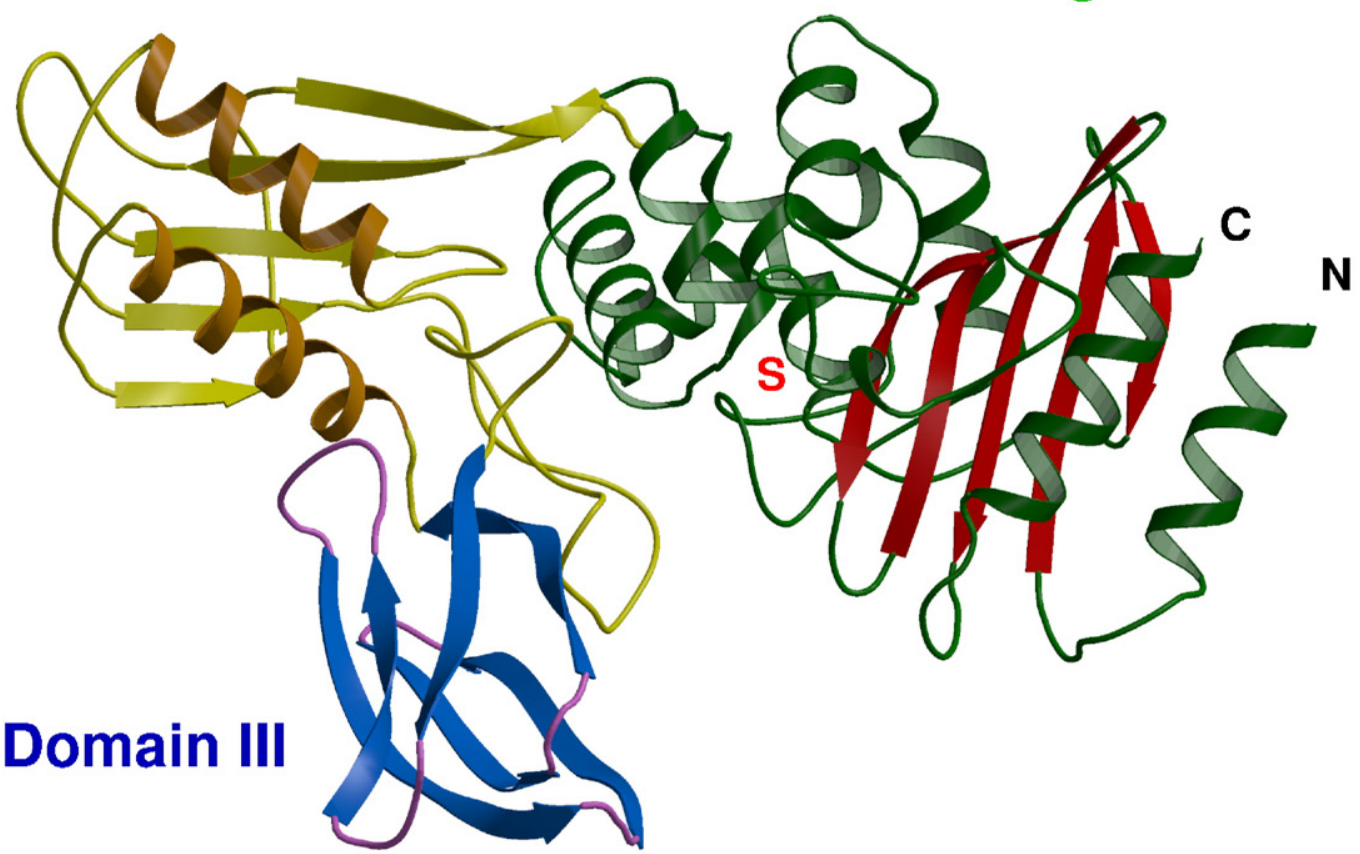

Figure 1 (legend on page 532) 

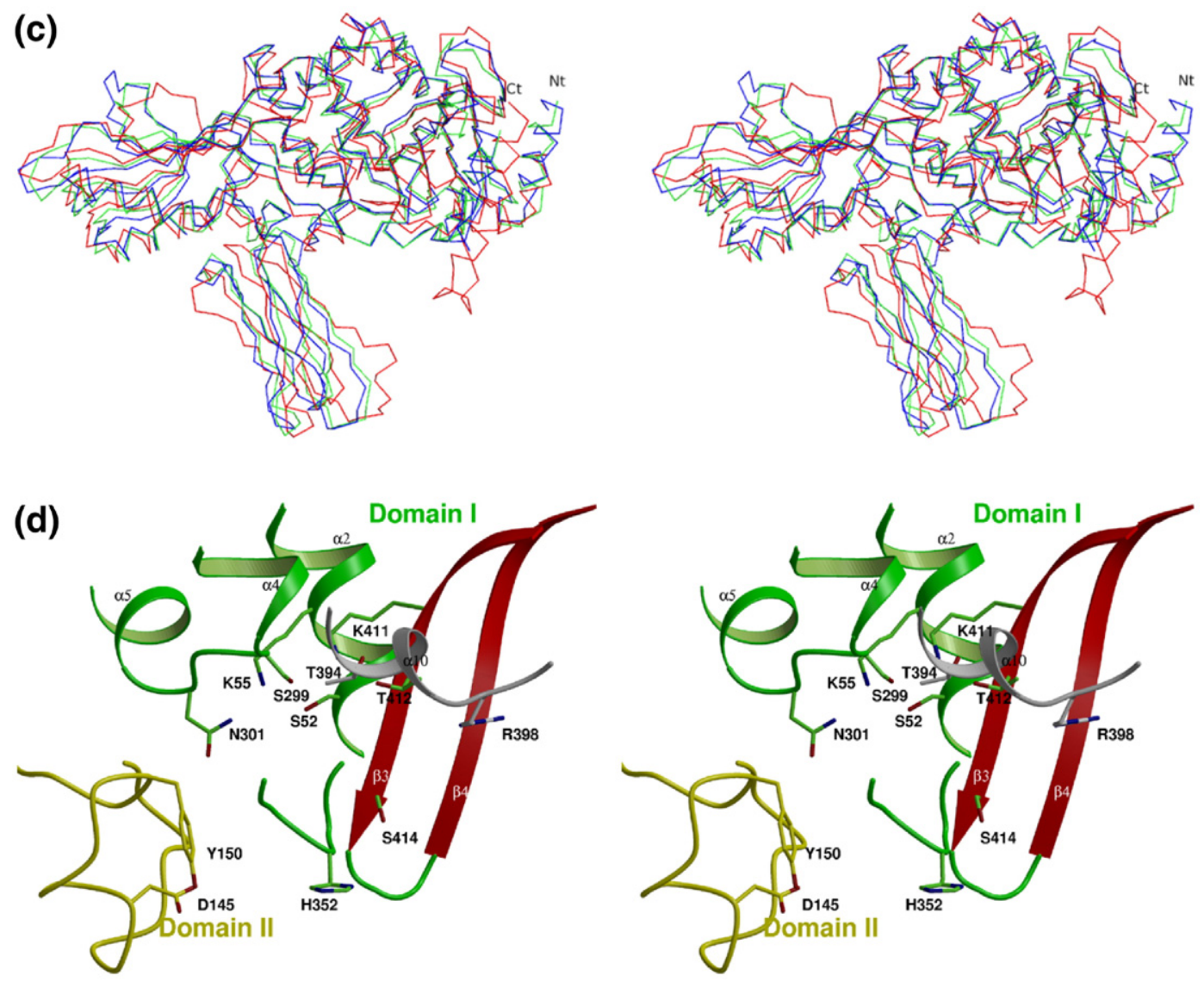

Figure 1 (legend on next page)

(KTG) at positions $411-413$ in the mature protein. The PBP4a penicillin-binding domain is composed of residues 5 to 68 and 288 to 462 . The first four Nterminal amino acid residues could not be localized in the electron density maps. The main differences between the fold of this domain and that of class A $\beta$-lactamases or E. coli PBP5 arise in the surroundings of the active site. Of note are the absence of the $\Omega$-loop and the replacement of the variable loop of class A $\beta$-lactamases (a loop inserted between motifs 1 and 2, which bounds one side of the active site) by the insertion of domains II and III. Compared to the R39 DD-peptidase and E. coli PBP4, 6,17 the positioning of the PBP4a active site residues is nearly identical with only minor differences affecting the very fine structure of the binding cavity.

\section{Domain II}

Domain II (residues 69-168 and 244-287) is a five stranded $\beta$-sheet with two helices packed on one side. The sheet is composed of four parallel and one antiparallel strands. The topology as well as the three-dimensional arrangement of this domain correspond to one-half of a Rossmann fold. As mentioned for R39 and E. coli PBP4, no known protein is structurally close to this domain. PBP4a domain II lacks sequence similarity with any topologically similar proteins.
A long unstructured loop (130-156) extends out of domain II. It packs onto the penicillin-binding domain, with residues Pro142, Asp145, and Tyr150 being part of a specific pocket in the bottom of the active site. On the opposite side of domain II, a series of lysine residues are protruding out of the core of the protein and seven of them (Lys83, Lys85, Lys86, Lys114, Lys119, Lys122 and Lys265) form a positively charged surface on the side opposite to the interface with the penicillin-binding domain (Figure 1(e)). A similar positive surface exists in R39, although involving other residues, but not in E. coli PBP4.

\section{Domain III}

Domain III (residues 169-243) is inserted into domain II and is composed of two three-stranded $\beta$ sheets facing each other. Few structural differences are observed between this domain and its equivalent in the R39 DD-peptidase. ${ }^{6}$ Differences are more pronounced with E. coli PBP4 although the general fold is similar. A DALI search with residues 169-243 did not yield any structurally similar protein.

\section{PBP4a-D- $\alpha$-aminopimelyl- $\varepsilon-D-a l a n i n e ~ c o v a l e n t$ adduct}

The crystal used for soaking was grown under crystallization conditions different from those that 
(e)

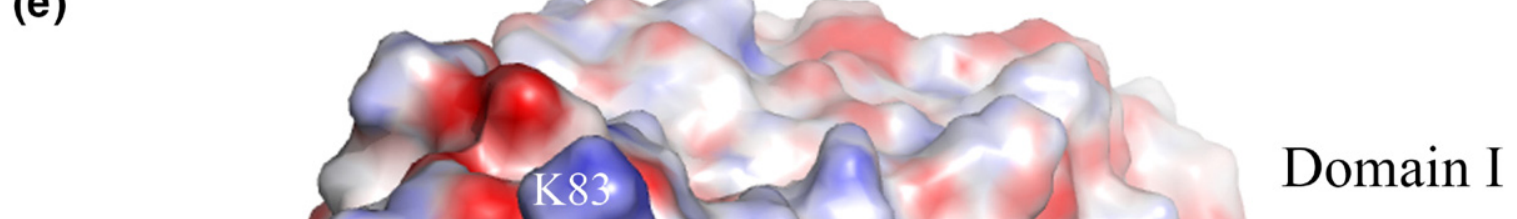

Domain II
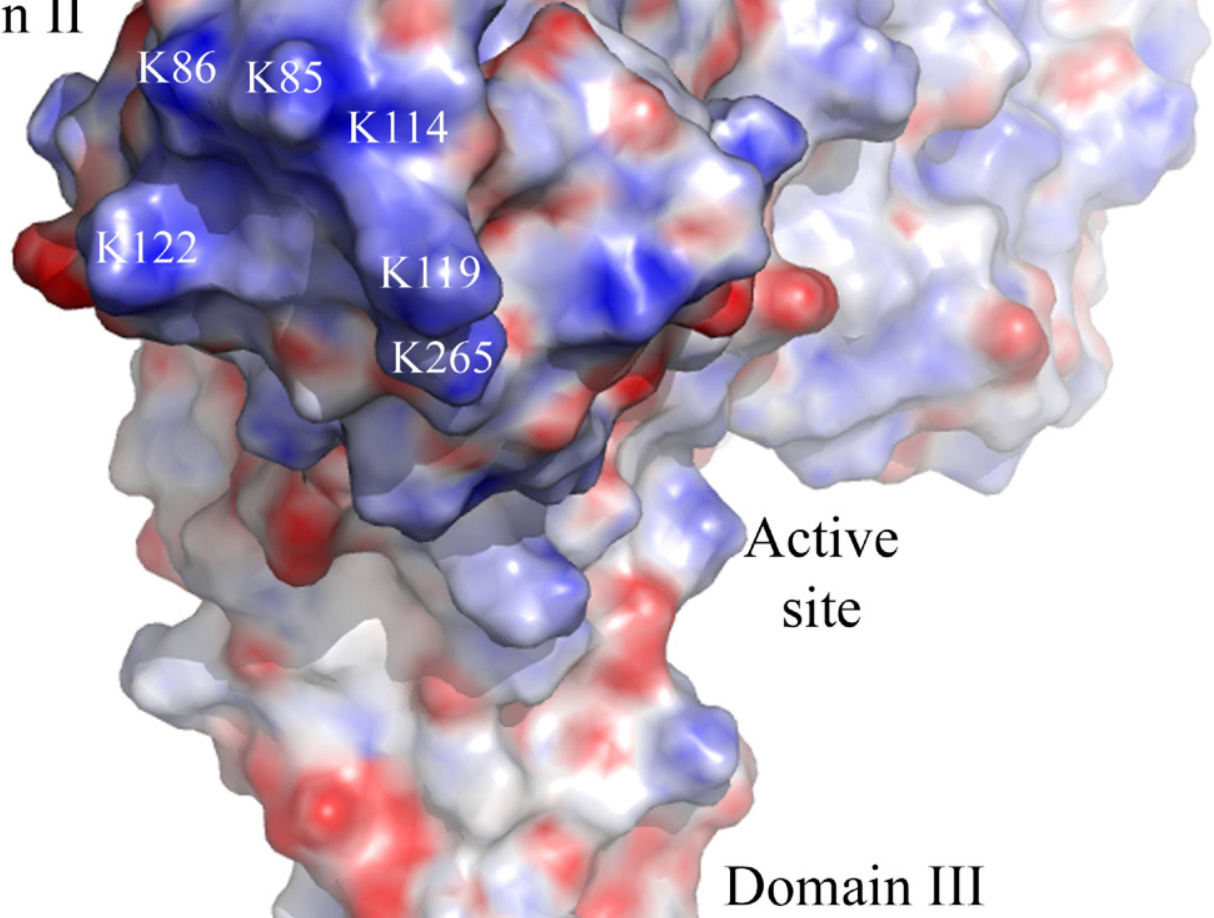

Figure 1. Structure of B. subtilis PBP4a. (a) Sequence alignment of PBP4a with three representatives of PBPs-C1. Active site catalytic residues of PBPs are colored red. Domains I, II and III are underlined by a green filled line, a yellow filled line and a blue filled line, respectively. Secondary structures are colored as in (b). (b) View of the overall structure of PBP4a showing the penicillin-binding domain in green and red, domain II in yellow and brown and domain III in blue and pink. The position of the active site serine is indicated by a red S. (c) Stereo view of the superposition of the $C^{\alpha}$ trace of $B$. subtilis PBP4a (green), Actinomadura R39 DD-peptidase (blue) and E. coli PBP4 (red). (d) Stereo view of the active site. The secondary structures are colored as in (b). The helix bearing residues Thr394 and Arg398 is grey for clarity. (e) Electrostatic potentials (red, negative; blue, positive) of PBP4a showing the basic surface in domain II. This view is approximately $75^{\circ}$ from (b).

provided the crystal used to solve the native structure. The absence of reproducibility of crystal growth with previous crystallization conditions might be related to a different purification protocol. The space group of the new crystal form is $P 2_{1} 2_{1} 2_{1}$ with two molecules per asymmetric unit. The structure of PBP4a under this crystalline form is identical to the structure solved in space group $P 3_{2} 12$ (rmsd $=0.516 \AA$ ) although some loops are slightly displaced, allowing the packing in space group $P 2{ }_{1} 22_{1}$. Many surface lysine side-chains are unresolved in the electron density maps.
Transpeptidation depends on the availability of a free amine acceptor and thus depends on the acceptor concentration. Inclusion of a high concentration of D-alanine $(900 \mathrm{mM})$ in the crystal soaking solution led to the trapping of an acyl-enzyme adduct and a D-alanine. The electron density in the active site of PBP4a can be attributed to an acylenzyme adduct between PBP4a and a D- $\alpha$-aminopimelyl- $\varepsilon$-D-alanine dipeptide and an unbound Dalanine, i.e. the products of acylation of PBP4a by $D-\alpha$-aminopymelyl- $\varepsilon$-D-alanyl-D-alanine with the release of a D-alanine (Figure 2(a) and (b)). The 
density is better defined in monomer B that we describe hereafter. The electron density is continuous between the active serine and the peptide and density is clearly defined for the ammonium carboxylate terminal group of the diaminopimelic acid. The methylene groups of the diaminopimelic acid side-chain are not well resolved and density appears progressively by lowering the $F_{\mathrm{o}}-F_{\mathrm{c}}$ omitmap contour level. The side-chain does however run through a hydrophobic cleft bounded by the sidechains of Tyr150, Ile350 and Leu415. The interpeptidic amide group is hydrogen bonded to the sidechain of Asn301 and to the backbone carbonyl of Ser414. The penultimate D-alanine carbonyl oxygen lies in the oxyanion hole, at $2.8 \AA$ and $2.7 \AA$ from Ser52 and Ser414 backbone nitrogen atoms, respectively. The penultimate D-alanine methyl group oc- cupies a hydrophobic pocket adjacent to the sidechain of Ile350 and $C^{\beta}$ of Ser348. A well defined methyl pocket has previously been identified on the R61 DD-peptidase. ${ }^{15}$

At the bottom of the active site, residues Pro142, Asp145, His352, and Ser416 form a pocket that accommodates the terminal $\mathrm{H}_{3} \mathrm{~N}^{+}-\mathrm{CH}-\mathrm{COO}^{-}$- group of the aminopimelic acid (Figure 2(c)). The amine nitrogen of the aminopimelic acid is at $2.9 \AA$ from Asp145. An aspartic acid lies at the same position in the R39 and E. coli PBP4 active sites and this residue is conserved in many PBPs-C1. A salt-bridge is formed between the $\alpha$-carboxylate of the aminopimelic acid and His352. In R39 and PBP4, the guanidinium group of an arginine lies at the same position as the imidazole group of His352 of PBP4a. In the primary structure of most PBPs-C1, this residue is a histidine

(a)
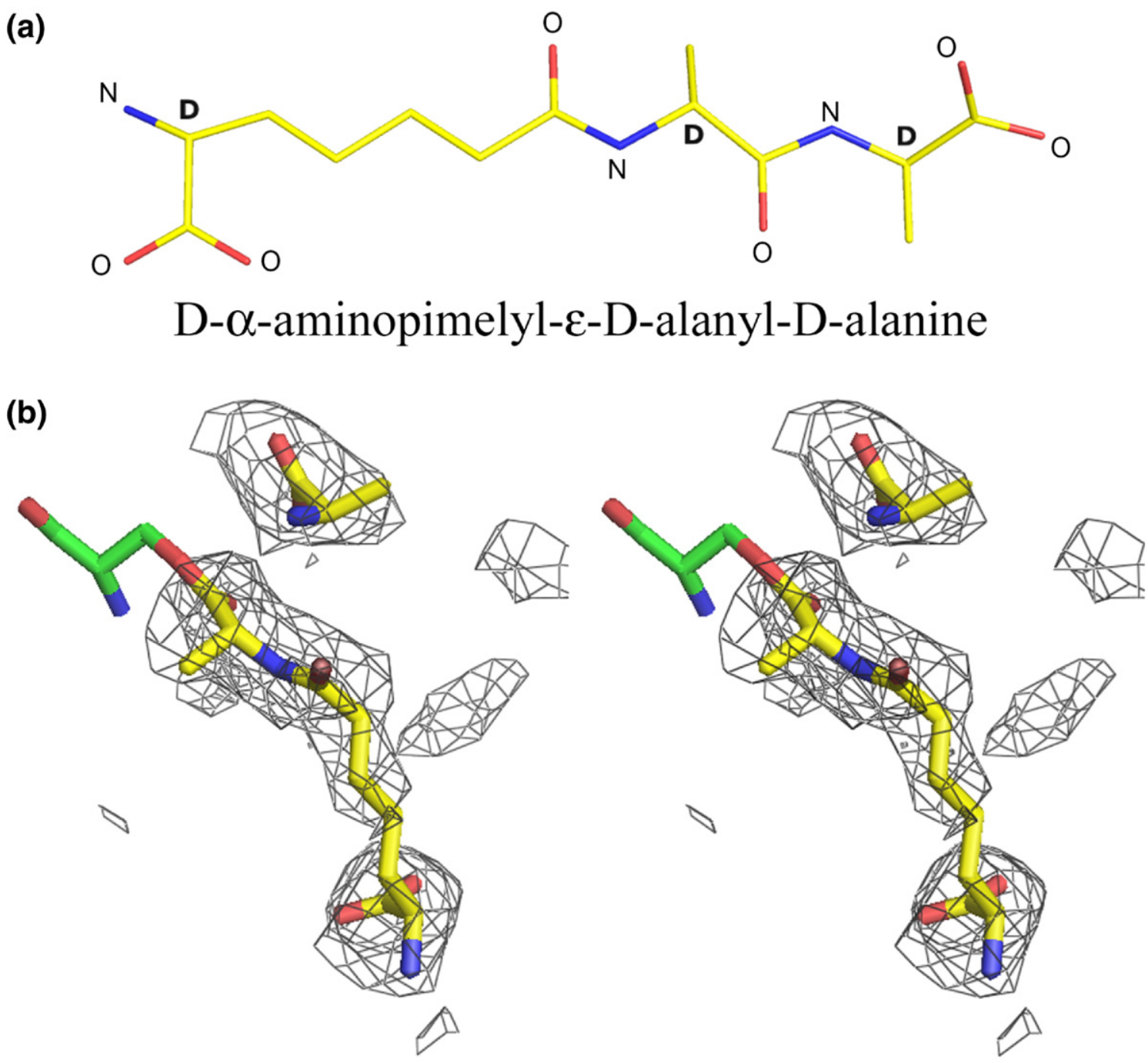

Figure 2. Structure of the adduct between B. subtilis PBP4a and D- $\alpha$-aminopymelyl- $\varepsilon$-D-alanine. (a) Structure of D- $\alpha$ aminopymelyl- $\varepsilon$-D-alanyl-D-alanine. Carbon atoms are colored yellow, oxygen atoms are colored red and nitrogen atoms are colored blue. (b) Stick representation of the D- $\alpha$-aminopymelyl- $\varepsilon$-D-alanine dipeptide covalently bonded to the active serine (shown in green) and the unbound D-alanine present in the active site. The omit map $F_{\mathrm{o}}-F_{\mathrm{c}}$ is calculated without the ligand and is cut at $1.5 \sigma$. (c) Surface representation of PBP4a and stick representation of the binding pocket specific to the diaminopimelic acid of the B. subtilis peptidoglycan stem peptide shown in cyan. The D- $\alpha$-aminopymelyl- $\varepsilon$-D-alanine dipeptide and the D-alanine are colored yellow and the active serine is colored green. 


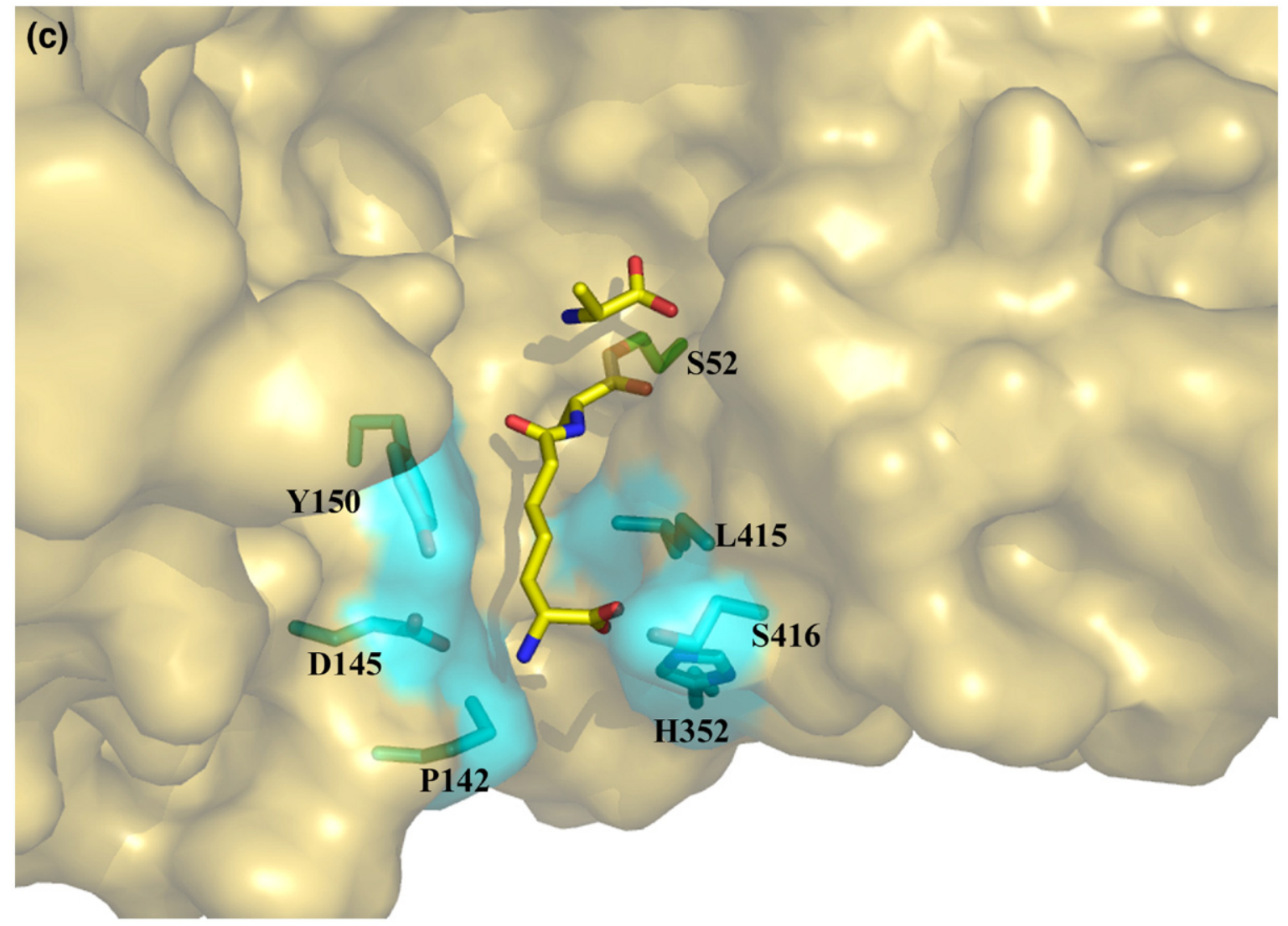

Figure 2 (legend on previous page)

or an arginine. The aminopimelic carboxylate is also hydrogen-bonded to the Ser416 hydroxyl group, another widely conserved group in PBPs-C1.

Because of the modest resolution, we have fitted the D-alanine in the density following chemical intuition, i.e. the carboxylate oriented towards the hydroxyl group of Thr412, and the amine towards Ser299 $\mathrm{O}^{\gamma}$. The final $2 F_{\mathrm{o}}-F_{\mathrm{c}}$ and $F_{\mathrm{o}}-F_{\mathrm{c}}$ maps are in agreement with the initial orientation of the Dalanine. The density of the initial $F_{\mathrm{o}}-F_{\mathrm{c}}$ map, calculated with protein coordinates, is too elongated and flat for a sulfate ion. After refinement with a sulfate ion instead of D-alanine, the $F_{\mathrm{o}}-F_{\mathrm{c}}$ map is worse than with the D-alanine, i.e. some positive and negative densities appear around the sulfate that are absent when using a D-alanine. Although map interpretation at $2.8 \AA$ remains rather subjective, maps look better with a D-alanine than with a sulfate ion and this is valuable in both monomers. The distance between the D-alanine amine and Ser299 (from the SxN motif) hydroxyl group is $2.7 \AA$, and the distance between the amine and the active serine Ser52 $\mathrm{O}^{\gamma}$ is $3.2 \AA$. The distance between the latter two serine $\mathrm{O}^{\gamma}$ atoms is $3.1 \AA$. The amine is placed close to an attack trajectory towards the acylenzyme carbonyl carbon atom, $3.1 \AA$ distant.

$\mathrm{D}-\alpha$-Aminopymelyl- $\varepsilon$-D-alanyl-D-alanine is a substrate for PBP4a $\left(k_{\text {cat }} / K_{\mathrm{m}}=2.2 \times 10^{4} \mathrm{M}^{-1} \mathrm{~s}^{-1}\right.$; $\left.K_{\mathrm{m}}>1 \mathrm{mM} ; k_{\text {cat }}>22 \mathrm{~s}^{-1}\right)$ but individual parameters of the reaction $\left(k_{2}\right.$ and $\left.k_{3}\right)$ were not measured. The accumulation of an acyl-enzyme in the crystal indicates that, within the conditions of the experiment, the deacylation step is rate-limiting in the DDcarboxypeptidation reaction catalyzed by PBP4a. In the R61 DD-peptidase, the crystallographic result revealed the products of reaction in the active site without any covalent bond between the active site serine and the penultimate D-alanine. ${ }^{15}$

The residues involved in the binding of the aminopimelic acid (Asp145, His352 and Ser416) are usually conserved among PBPs-C1. A specific binding pocket, different from that of PBP4a, has been highlighted in the PBP-C2 R61 DD-peptidase in which this subsite is responsible for the high affinity of R61 for the cell wall peptidoglycan of Streptomyces, which contains LL-diaminopimelic acid instead of meso-diaminopimelic acid and a glycine residue in the interpeptidic bridge. ${ }^{15}$

In the complex of PBP5 with Boc- $\gamma$-D-Glu-L-Lys (Cbz)-D-boroAla (pdb code 1Z6F), ${ }^{11}$ the boronyl group mimics the transition state of a PBP-catalyzed deacylation reaction. One of its oxygen atoms lies in the oxyanion hole similarly to the D-alanine oxygen of the serine bound dipeptide. The position of the other oxygen, mimicking the attacking water molecule in a carboxypeptidation reaction, is close to the position of the amine group of the unbound D-alanine in the PBP4a adduct. The methyl groups of the aminopimelic acid side-chain (or its mimicking equivalent in PBP5) are roughly identically posi- 
tioned in both structures. The main difference is the apparent absence in PBP5 of a pocket binding specifically the $\mathrm{H}_{3} \mathrm{~N}^{+}-\mathrm{CH}-\mathrm{COO}^{-}$group of the aminopimelic acid as seen in PBP4a.

\section{Sensitivity to $\beta$-lactam}

PBP4a is moderately sensitive to $\beta$-lactam antibiotics (Table 1). The fold of the active site of PBP4a is close to that of the R39 DD-peptidase ${ }^{6}$ and E. coli $\mathrm{PBP} 4{ }^{17}$. The difference between the acylation rate constants by benzylpenicillin of these proteins is striking because no clear structural characteristic in or near the active site can account for this difference in sensitivity to $\beta$-lactams.

\section{Discussion}

The crystal structures of PBP4a and its adduct with $D$ - $\alpha$-aminopimelyl- $\varepsilon$-D-alanyl-D-alanine provide information about the specificity of the enzymatic activity of PBPs-C1 that are described as DD-carboxypeptidases, DD-transpeptidases or DD-endopeptidases. ${ }^{18-23}$ A DD-transpeptidase activity has been observed in vitro but it is unlikely the physiological role of these PBPs as clearly shown for the E. coli PBP4 by Korat et al. who compared the peptidoglycan composition of a PBP4 overproducing strain with that of the reference strain. ${ }^{22}$ The DD-carboxypeptidase activity of PBP4a has been demonstrated on $N^{\alpha}$-acetyl-L-Lys-D-Ala-D-Ala. ${ }^{18}$

\section{DD-Carboxypeptidase/endopeptidase versus transpeptidase activity}

The adduct between PBP4a and D- $\alpha$-aminopimelyl- $\varepsilon$-D-alanine with the release of a D-alanine confirms the DD-carboxypeptidase activity of PBP4a although it could also represent the acyl enzyme intermediate for the transpeptidation reaction. The D-alanine released during the carboxypeptidation reaction could play the role of acceptor in a transpeptidation reaction that would regenerate the D- $\alpha$-aminopimelyl- $\varepsilon$-D-alanyl-D-alanine tripeptide. The transpeptidation reaction depends on acceptor concentration and, as we were interested in obtaining the product of the transpeptidase activity of PBP4a, we added a high concentration of D-alanine in the crystal soaking solution. This led to the trapping of the carboxypeptidase-acylation reaction products but not to the transpeptidase reaction

Table 1. Second order rate constants for acylation of PBPs-C1 by benzylpenicillin

\begin{tabular}{lcc}
\hline Enzyme & $k_{2} / K_{\mathrm{m}}\left(\mathrm{M}^{-1} \mathrm{~s}^{-1}\right)$ & Reference \\
\hline B. subtilis PBP4a & 1400 & 18 \\
R39 DD-peptidase & $3 \times 10^{5}$ & 47 \\
E. coli PBP4 & 7000 & 48 \\
N. gonorrhoeae PBP3 & $(2-7.7) \times 10^{5}$ & 19 \\
P. mirabilis PBP4 & $(2-3) \times 10^{5}$ & 48 \\
\hline
\end{tabular}

product, indicating that $\mathrm{PBP} 4 \mathrm{a}$ is rather a DDcarboxypeptidase or an endopeptidase than a DDtranspeptidase, or that D-alanine is not a good acceptor.

In a transpeptidation reaction, D- $\alpha$-aminopymelyl-e-D-alanyl-D-alanine could itself have played the role of the acceptor because in B. subtilis, the crosslinking of the peptidoglycan chains occurs between the penultimate alanine carbonyl group of a donor strand and the terminal amine group on the D center of the meso-diaminopimelic acid of an acceptor strand. The diaminopimelic acid ammonium-carboxylate terminal group in $\mathrm{D}$ configuration is structurally equivalent to the leaving D-alanine, unlike the situation in R61 where the glycyl group bridging the donor and acceptor peptidoglycan stem peptides is different from the leaving Dalanine. ${ }^{24}$ In fact, we do see what is apparently a specific binding site for a $\mathrm{D}$ - $\alpha$-aminopimelyl- $\varepsilon$-Dalanyl donor, analogous to what is observed in R61 where there is a specific site for a glycyl-L- $\alpha$ aminopimelyl- $\varepsilon$-D-alanyl donor. Following the release of D-alanine, the free amine group of an acceptor diaminopimelic acid should be able to attack the ester bond to generate a $D$ - $\alpha$-aminopimelyl- $\varepsilon$-D-alanyl-D- $\alpha$-aminopimelic acid. Electron density accounting for an acceptor peptide could not be observed, indicating that the affinity for $D-\alpha-$ aminopimelyl- $\varepsilon$-D-alanyl-D-alanine of a putative acceptor site is much lower than the donor binding site.

\section{Catalytic mechanism}

Residues involved in the mechanisms of acylation and deacylation by PBPs are not clearly identified. Acylation has been widely discussed in the framework of serine $\beta$-lactamases and more briefly for PBPs. ${ }^{13,25}$ The mechanism encompassing the role of the lysine 55 as the general base is the most likely. ${ }^{4,9-11}$ The lysine needs to be unprotonated to abstract the proton from the active serine and, as seen in many crystallographic structures of PBPs, the proximity of Lys55 to Ser52 supports this idea. The abstracted proton is then transferred to the C-terminal D-alanine to complete acylation. It has been suggested that the proton abstraction could be accomplished by the substrate carboxylate. ${ }^{12}$ Alternatively, a concerted one-step process where the covalent bond formation between the penultimate D-alanine and the active serine Ser52 is concomitant with the transfer of the Ser52 hydroxyl proton directly to Ser299 and with the transfer of the Ser299 hydroxyl proton to the leaving D-alanine nitrogen is possible. ${ }^{26}$

A clear and extensive discussion of the deacylation mechanism is provided by Nicola et al. ${ }^{11}$ The unbound D-alanine present in the active site of the complex structure of PBP4a represents the acceptor in the deacylation step of a transpeptidation reaction. A proton cannot be directly removed from its amine group by Lys 55 because this residue is too far from it. On the reverse, Ser299 is close to the amine 
group and the network of hydrogen bonding interactions suggests that Lys411 could be the general base as proposed for PBP5. ${ }^{11}$ Lys411 would promote deacylation by polarizing Ser299, and Ser299 would directly polarize the acceptor amine group attacking the electrophilic carbonyl of the acyl-enzyme.

\section{DD-Carboxypeptidase versus endopeptidase or transpeptidase activity}

Some PBPs (e.g. E. coli PBP5) are strict DDcarboxypeptidases and are devoid of endopeptidase or transpeptidase activity. This may mean that they cannot accommodate a bulky side-chain rather than the ultimate D-alanine of the stem peptide. Steric interactions may be the source of this behaviour. Although most PBP backbones do not closely superimpose that of $\mathrm{PBP} 4 \mathrm{a}$ in the vicinity of the helix bearing Thr394, a threonine is frequently found in a position analogous to Thr394 in PBP4a. Thr394 $\mathrm{O}^{\delta}$ is hydrogen-bonded to Thr412 $\mathrm{O}^{\delta}$ and contributes to the hydrogen-bond network that strengthens the active site. In E. coli PBP5, a PBP devoid of endopeptidase activity, an arginine lies in the equivalent position and might impede the positioning of a longer chain than the methyl group of the ultimate D-alanine of the stem peptide. The presence of a smaller residue such as a threonine leaves some free space to accommodate the stem peptide of a second peptidoglycan chain. This feature could explain why PBPs-C1 can act as DD-endopeptidases whereas PBP5 exhibits preferentially a DD-carboxypeptidase activity. The E. coli PBP5, PBP6 and PBP6b DD-carboxypeptidases all have an arginine in that position whereas the E. coli PBP4 and PBP7 DDendopeptidases have a threonine or a serine. The vast majority of PBPs-A and PBPs-B have a threonine or a serine in the equivalent position and it could be tentatively concluded that a bulky sidechain is unfavorable for DD-transpeptidase or DDendopeptidase activities. PBPs with such a bulky side-chain might therefore be strict DD-carboxypeptidases whereas the others could behave either as DD-transpeptidases or DD-endopeptidases or both. Unfortunately, the DD-carboxypeptidase, DD-transpeptidase and DD-endopeptidase activities of most PBPs are not sufficiently documented for this conclusion to be definite.

\section{Role of PBP4a}

The large number of PBPs in B. subtilis makes it difficult to assign a precise role to each of them. For example, the DD-carboxypeptidase activity is mediated by a series of low molecular mass PBPs, which could contribute to peptidoglycan maturation and maintenance of the bacterial morphology. Nevertheless, each PBP could exert its activity at a peculiar moment of the life cycle to fulfil a precise role in peptidoglycan remodelling. ${ }^{27}$

A series of tracks to determine the role of PBPs in $B$. subtilis have been explored. Green fluorescent protein (GFP) fusions were constructed with all 11 PBPs produced during vegetative growth. ${ }^{28} \mathrm{PBP} 4 \mathrm{a}$ and PBP3 appeared in distinct foci to the lateral wall. A 3-D reconstruction of fluorescent signals of GFPPBP4a and GFP-PBP3 revealed short arcs at the cell periphery partially co-localizing with the $\mathrm{Mbl}$ cytoskeleton. However, similar localization patterns were observed in an mbl knock-out strain. PBP3 and PBP4a seemed actively recruited to the lateral wall and the authors of this study suggested a role for these PBPs (as well as for PBP5 and possibly PBP4) in lateral cell wall synthesis of this rod-shaped organism. However, the absence of PBP3, PBP4 or PBP4a had no major effects on $B$. subtilis cell morphology. ${ }^{29}$ Furthermore, the bacteria from the genus Neisseria, which contain a reduced number of PBPs (only four in N. meningitidis and in N. gonorrhoeae), do possess a low molecular mass PBP-C1. These bacteria are cocci and PBPs-C1 cannot therefore be involved in cell wall elongation, at least in this case.

Low molecular mass PBPs, and more particularly PBPs-C1, are generally considered as involved in peptidoglycan maturation. E. coli PBP4 is indirectly involved in cell morphology, ${ }^{30}$ in daughter cells separation, ${ }^{31}$ and could be implicated in biofilm formation. ${ }^{32}$ In contrast to most other PBPs, which are strongly anchored in the membrane, PBPs-C1 are soluble or loosely bound to the membrane ${ }^{19,20,33,34}$ and based on hydrophobic moment analyses, it was suggested that the interaction of E. coli PBP4 with the membrane is of electrostatic nature between the cationic residues of a C-terminal peptide and the negatively charged moieties of the membrane. ${ }^{34}$ Such interactions are weak and give a limited level of membrane penetration. This type of anchoring is unlikely for B. subtilis PBP4a, which possesses more acidic than basic amino acids in its 21-residue C-terminal peptide. Furthermore, the removal of its C-terminal $\alpha$-helix did not prevent PBP4a from being associated with the membrane when produced in E. coli. ${ }^{33}$ PBP4a could interact with a protein located in the membrane through proteinprotein interactions or could interact directly with the membrane via the charged surface of domain II. The charge distribution on the surface of domain II is remarkable. Seven lysine residues protrude from the core of domain II and form a positively charged surface on the side opposite to the interface with the penicillin-binding domain (Figure 1(e)). These lysine residues are not conserved in the primary structure of PBPs-C1 and this feature might be unique to B. subtilis PBP4a. Nevertheless, due to a great variability in the sequences of PBPs-C1 in this region, a positive surface could result from other positively charged residues: four arginine residues and a lysine were found to define such a surface in the R39 DD-peptidase. ${ }^{6}$

Another possibility is that PBP4a could be localized on the outer face of the peptidoglycan and would therefore exert its endopeptidase activity on the external face of the peptidoglycan. The positively charged surface found on the domain II of PBP4a could interact with teichoic acids or teichuro- 
nic acids, anionic polymers covalently bonded to peptidoglycan and very abundant in the $B$. subtilis wall, a situation that also prevails in the Actinomycetales ${ }^{35}$ and could explain the similar positively charged surface found in the DD-peptidase from Actinomadura R39.

\section{Experimental Procedures}

\section{PBP4a purification}

The purification protocol previously described ${ }^{18}$ has been simplified to a single step of chromatography on Heparin-Sepharose CL-6B (Amersham Biosciences, Roosendal, The Netherlands) a highly sulfated glycosaminoglycan. Heparin-Sepharose CL-6B (10 g) was equilibrated in $45 \mathrm{ml}$ of $0.1 \mathrm{M}$ Tris- $\mathrm{HCl}$ buffer $(\mathrm{pH} \mathrm{7.8)}$ ) and poured in a $2.4 \mathrm{~cm}$ diameter column. The recombinant PBP4a producing cells were harvested from 11 of culture, resuspended in $50 \mathrm{ml}$ of $0.1 \mathrm{M}$ Tris- $\mathrm{HCl}(\mathrm{pH} 7.8)$ and lysed in a French Press (SLM-AMINCO) at $20 \mathrm{kPsi}$. The supernatant from a $30 \mathrm{~min}$ centrifugation at $25,000 \mathrm{~g}$ was loaded onto the column. After the column was washed with the same buffer, the ionic strength was increased in stages: 0.2 and $0.4 \mathrm{M} \mathrm{KCl}$ was successively added to the initial buffer and washes were carried out until the absorbance at $280 \mathrm{~nm}$ of the eluate was negligible. Fractions of $8 \mathrm{ml}$ were collected when buffers containing $0.6 \mathrm{M}$ and then $0.8 \mathrm{M} \mathrm{KCl}$ were applied to the column. PBP4a was detected on the basis of its hydrolyzing activity on the $N^{\alpha}$-acetyl-L-lys-D-ala-D-ala substrate. The active fractions were concentrated, supplemented with $0.02 \%(\mathrm{w} / \mathrm{v})$ sodium azide and stored at $4{ }^{\circ} \mathrm{C}$ without dialysis. $15 \mathrm{mg}$ of PBP4a with $98 \%$ purity were recovered from a 11 culture.

\section{Crystallization and data collection of the native enzyme}

The initial crystallization conditions were established using sparse matrix sampling ${ }^{36}$ with the hanging-drop vapor diffusion method ${ }^{37}$ at $293 \mathrm{~K}$ using the different commercially available crystallization kits (Hampton Research, Emerald Biostructures). Drops were prepared by mixing $2 \mu \mathrm{l}$ of protein solution (at a concentration of $20 \mathrm{mg} \mathrm{ml}^{-1}$ in $0.1 \mathrm{M}$ Tris- $\left.\mathrm{HCl}(\mathrm{pH} 7.8), 0.8 \mathrm{M} \mathrm{KCl}\right)$ with $2 \mu \mathrm{l}$ of reservoir solution. Crystals of small size and poor quality were obtained under several conditions. The best ones were obtained from condition number 7 of the PEG/ Ion Screen formulations from Hampton Research. They grew as small prisms from 20\% (w/v) PEG 3350, $0.2 \mathrm{M}$ $\mathrm{CaCl}_{2}$, (pH 5.1).

Before X-ray data collection, the PBP4a crystal was flash frozen in liquid nitrogen after rapid soaking in a cryoprotectant solution containing $40 \%(\mathrm{v} / \mathrm{v})$ glycerol in the crystallization buffer. Native data were collected at $100 \mathrm{~K}$ on an ADSC Quantum 4R CCD detector at a wavelength of $0.9763 \AA$ on beamline ID29 at the European Synchrotron Radiation Facility (ESRF, Grenoble, France). Intensities were indexed and integrated using MOSFLM version $6.01 .^{38}$ The crystal, belonging to space-group $P 3_{2} 12$ with unit cell dimensions $a=b=67.41 \AA$, $c=228.46 \AA$, diffracted to about $2.1 \AA$ resolution. The scaling of the intensity data was accomplished with SCALA of the CCP4 program suite ${ }^{39}$ and all corresponding statistics are given in Table 2.

\section{Phasing procedure and structure refinement}

The phasing procedure for solving the PBP4a crystal structure was the molecular replacement method. The rotational and translational parameter investigations were performed with $\mathrm{AMoRe}^{40}$ using as search model the coordinates of the DD-peptidase from Actinomadura R39 (PDB 1W79). Subsequent refinement was carried out using $\mathrm{CNS}^{41}$ and manual model fitting with Turbo-Frodo. ${ }^{42}$ The PBP4a structure was refined to $2.1 \AA$ resolution and the statistics of refinement are summarized in Table 2.

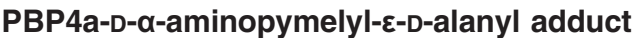

D- $\alpha$-Aminopymelyl- $\varepsilon$-D-alanyl-D-alanine was prepared as described. ${ }^{16}$ Due to a new purification protocol, crystals obtained previously could not be reproduced and a new screening led to new crystallization conditions. A crystal of the PBP4a was obtained by mixing $2 \mu 1$ of protein solution (at a concentration of $22 \mathrm{mg} \mathrm{ml}^{-1}$ in $0.1 \mathrm{M}$ Tris$\mathrm{HCl}$ (pH 7.8), $0.8 \mathrm{M} \mathrm{KCl}, 20 \mathrm{mM} \mathrm{CoCl}_{2}, 10 \mathrm{mM} \mathrm{NaN}_{3}$ ) with $2 \mu \mathrm{l}$ of reservoir solution (20\% PEG $5000 \mathrm{MME}, 0.1 \mathrm{M}$ Hepes $\mathrm{pH} 7$ ). Soaking of the PBP4a crystal was performed by adding slowly a solution of $\mathrm{D}-\alpha$-aminopymelyl- $\varepsilon$-Dalanyl-D-alanine mixed with D-Ala to final concentrations of $23.4 \mathrm{mM}$ and $900 \mathrm{mM}$, respectively. Before X-ray data collection, the PBP4a crystal was cryoprotected with $100 \%$ MPD. Data were collected at $100 \mathrm{~K}$ on a MAR CCD detector at beamline BM30a at ESRF. Intensities were indexed and integrated using MOSFLM version 6.01. ${ }^{38}$ The crystal, belonging to space-group $P 2{ }_{1} 2_{1} 2_{1}$ with unit cell dimensions $a=73.86 \AA, b=77.59 \AA, c=164.74 \AA$, diffracted to about $2.8 \AA$ resolution. Data were scaled with SCALA of the CCP4 program suite ${ }^{39}$ and all corresponding statistics are given in Table 2. Figures were prepared with Pymol, ${ }^{43}$ Molscript $^{44}$ Bobscript $^{45}$ and Raster3D. ${ }^{46}$

Table 2. Data collection, phasing and refinement statistics

\begin{tabular}{|c|c|c|}
\hline & Apo-enzyme & Complex \\
\hline PDB code & 1W5D & 202J9P \\
\hline Space group & $\mathrm{P}_{2} 12$ & $P 2_{1} 2_{1} 2_{1}$ \\
\hline \multicolumn{3}{|l|}{ Cell dimensions } \\
\hline$a(\AA)$ & 67.4 & 73.9 \\
\hline$b(\AA)$ & 67.4 & 77.6 \\
\hline$c(\AA)$ & 228.5 & 164.7 \\
\hline Wavelength $(\AA)$ & 0.9763 & 1.0 \\
\hline Resolution range $(\AA)^{\mathrm{a}}$ & $20-2.1(2.21-2.1)$ & $24.6-2.8(2.95-2.8)$ \\
\hline Unique reflections & 34,499 & 17,249 \\
\hline Redundancy ${ }^{\mathrm{a}}$ & $5.8(4.4)$ & $5.5(5.4)$ \\
\hline Completeness (\%) ${ }^{\mathrm{a}}$ & $97.7(85.6)$ & $91.0(93.0)$ \\
\hline$I / \sigma(I)^{\mathrm{a}}$ & $13.1(4.5)$ & $11.2(2.4)$ \\
\hline$R_{\text {sym }}(\%)^{\mathrm{a}}$ & $8.1(28.7)$ & $15.1(58.0)$ \\
\hline \multicolumn{3}{|l|}{ Refinement } \\
\hline Resolution range $(\AA)$ & $20-2.1$ & $20-2.8$ \\
\hline$R_{\text {cryst }} / R_{\text {free }}(\%)$ & $22.4 / 26.8$ & $20.8 / 29.0$ \\
\hline Number of protein atoms & 3462 & 6922 \\
\hline $\begin{array}{l}\text { Number of water } \\
\text { molecules }\end{array}$ & 359 & - \\
\hline \multicolumn{3}{|l|}{ r.m.s. deviations } \\
\hline Bond lengths $(\AA)$ & 0.006 & 0.007 \\
\hline Bond angles $\left({ }^{\circ}\right)$ & 1.3 & 1.4 \\
\hline
\end{tabular}

${ }^{\text {a }}$ Statistics for the highest resolution shell are given in parentheses. 


\section{Protein Data Bank accession codes}

The atomic coordinates for the crystal structure of this protein have been deposited in the RCSB Protein Data Bank and are available under the accession code 1W5D (native) and 2J9P (adduct).

\section{Acknowledgements}

We thank the staff of beamlines ID29 and BM30a at ESRF for assistance in X-ray data collection. This work was supported in part by the Belgian Program on Interuniversity Poles of Attraction initiated by the Belgian State, Prime Minister's Office, Science Policy Programming (PAI 6/19), by the Fonds National de la Recherche Scientifique (IISN 4.4505.00, FRFC 9.45/9.99, FRFC 2.4.508.01.F, FRFC 9.4.538.03.F, FRFC 2.4.524.03) and the University of Liège (Fonds spéciaux, Crédit classique, 1999). C.D. is Chercheur Qualifié of the Fonds National de la Recherche scientifique (Brussels, Belgium) and S.P. is a fellow of the French "Fondation Recherche Médicale". J.W.A. and R.F.P. were supported by the National Institutes of Health (AI -17986).

\section{References}

1. van Heijenoort, J. (2001). Formation of the glycan chains in the synthesis of bacterial peptidoglycan. Glycobiology, 11, 25R-36R.

2. Goffin, C. \& Ghuysen, J. M. (1998). Multimodular penicillin-binding proteins: an enigmatic family of orthologs and paralogs. Microbiol. Mol. Biol. Rev. 62, 1079-1093.

3. Yanouri, A., Daniel, R. A., Errington, J. \& Buchanan, C. E. (1993). Cloning and sequencing of the cell division gene $p b p B$, which encodes penicillin-binding protein 2B in Bacillus subtilis. J. Bacteriol. 175, 7604-7616.

4. Nicholas, R. A., Krings, S., Tomberg, J., Nicola, G. \& Davies, C. (2003). Crystal structure of wild-type penicillin-binding protein 5 from Escherichia coli: implications for deacylation of the acyl-enzyme complex. J. Biol. Chem. 278, 52826-52833.

5. Morlot, C., Pernot, L., Le Gouellec, A., Di Guilmi, A. M., Vernet, T., Dideberg, O. \& Dessen, A. (2005). Crystal structure of a peptidoglycan synthesis regulatory factor (PBP3) from Streptococcus pneumoniae. J. Biol. Chem. 280, 15984-15991.

6. Sauvage, E., Herman, R., Petrella, S., Duez, C., Bouillenne, F., Frère, J. M. \& Charlier, P. (2005). Crystal structure of the Actinomadura R39 DD-peptidase reveals new domains in penicillin-binding proteins. J. Biol. Chem. 280, 31249-31256.

7. Kelly, J. A. \& Kuzin, A. P. (1995). The refined crystallographic structure of a DD-peptidase penicillin-target enzyme at $1.6 \AA$ Å resolution. J. Mol. Biol. 254, 223-236.

8. Fonzé, E., Vermeire, M., Nguyen-Distèche, M., Brasseur, R. \& Charlier, P. (1999). The crystal structure of a penicilloyl-serine transferase of intermediate penicillin sensitivity. The DD-transpeptidase of Streptomyces K15. J. Biol. Chem. 274, 21853-21860.
9. Gordon, E., Mouz, N., Duee, E. \& Dideberg, O. (2000). The crystal structure of the penicillin-binding protein $2 \times$ from Streptococcus pneumoniae and its acyl-enzyme form: implication in drug resistance. J. Mol. Biol. 299, 477-485.

10. Lim, D. \& Strynadka, N. C. (2002). Structural basis for the beta lactam resistance of PBP2a from methicillinresistant Staphylococcus aureus. Nature Struct. Biol. 9, 870-876.

11. Nicola, G., Peddi, S., Stefanova, M., Nicholas, R. A., Gutheil, W. G. \& Davies, C. (2005). Crystal structure of Escherichia coli penicillin-binding protein 5 bound to a tripeptide boronic acid inhibitor: a role for Ser-110 in deacylation. Biochemistry, 44, 8207-8217.

12. Ishiguro, M. \& Imajo, S. (1996). Modeling study on a hydrolytic mechanism of class A beta-lactamases. J. Med. Chem. 39, 2207-2218.

13. Macheboeuf, P., Contreras-Martel, C., Job, V., Dideberg, O. \& Dessen, A. (2006). Penicillin binding proteins: key players in bacterial cell cycle and drug resistance processes. FEMS Microbiol. Rev. 30, 673-691.

14. Ghuysen, J. M. (1991). Serine beta-lactamases and penicillin-binding proteins. Annu. Rev. Microbiol. 45, 37-67.

15. McDonough, M. A., Anderson, J. W., Silvaggi, N. R., Pratt, R. F., Knox, J. R. \& Kelly, J. A. (2002). Structures of two kinetic intermediates reveal species specificity of penicillin-binding proteins. J. Mol. Biol. 322, 111-122.

16. Anderson, J. W., Adediran, S. A., Charlier, P., NguyenDistèche, M., Frère, J. M., Nicholas, R. A. \& Pratt, R. F. (2003). On the substrate specificity of bacterial DDpeptidases: evidence from two series of peptidoglycan-mimetic peptides. Biochem. J. 373, 949-955.

17. Kishida, H., Unzai, S., Roper, D. I., Lloyd, A., Park, S. Y. \& Tame, J. R. (2006). Crystal structure of penicillin binding protein $4(d a c B)$ from Escherichia coli, both in the native form and covalently linked to various antibiotics. Biochemistry, 45, 783-792.

18. Duez, C., Vanhove, M., Gallet, X., Bouillenne, F., Docquier, J., Brans, A. \& Frère, J. (2001). Purification and characterization of PBP4a, a new low-molecularweight penicillin-binding protein from Bacillus subtilis. J. Bacteriol. 183, 1595-1599.

19. Stefanova, M. E., Tomberg, J., Olesky, M., Holtje, J. V., Gutheil, W. G. \& Nicholas, R. A. (2003). Neisseria gonorrhoeae penicillin-binding protein 3 exhibits exceptionally high carboxypeptidase and beta-lactam binding activities. Biochemistry, 42, 14614-14625.

20. Rousset, A., Nguyen-Distèche, M., Minck, R. \& Ghuysen, J. M. (1982). Penicillin-binding proteins and carboxypeptidase/transpeptidase activities in Proteus vulgaris $\mathrm{P} 18$ and its penicillin-induced stable L-forms. J. Bacteriol. 152, 1042-1048.

21. Yasukawa, H., Kuroita, T., Tamura, K. \& Yamaguchi, K. (2003). Identification of a penicillin-sensitive carboxypeptidase in the cellular slime mold Dictyostelium discoideum. Biol. Pharm. Bull. 26, 1018-1020.

22. Korat, B., Mottl, H. \& Keck, W. (1991). Penicillinbinding protein 4 of Escherichia coli: molecular cloning of the $d a c B$ gene, controlled overexpression, and alterations in murein composition. Mol. Microbiol. 5, 675-684.

23. Zhao, G. H., Duez, C., Lepage, S., Forceille, C., Rhazi, N., Klein, D. et al. (1997). Site-directed mutagenesis of the Actinomadura R39 DD-peptidase. Biochem. J. 327, 377-381.

24. Kumar, I. \& Pratt, R. F. (2005). Transpeptidation reactions of a specific substrate catalyzed by the 
Streptomyces R61 DD-peptidase: the structural basis of acyl acceptor specificity. Biochemistry, 44, 9961-9970.

25. Oliva, M., Dideberg, O. \& Field, M. J. (2003). Understanding the acylation mechanisms of active-site serine penicillin-recognizing proteins: a molecular dynamics simulation study. Proteins: Struct. Funct. Genet. 53, 88-100.

26. Dive, G. \& Dehareng, D. (1999). Serine peptidase catalytic machinery: cooperative one-step mechanism Int. J. Quant. Chem. 73, 161-174.

27. Young, K. D. (2003). Bacterial shape. Mol. Microbiol. 49, 571-580.

28. Scheffers, D. J., Jones, L. J. \& Errington, J. (2004). Several distinct localization patterns for penicillinbinding proteins in Bacillus subtilis. Mol. Microbiol. 51, 749-764.

29. Popham, D. L., Gilmore, M. E. \& Setlow, P. (1999). Roles of low-molecular-weight penicillin-binding proteins in Bacillus subtilis spore peptidoglycan synthesis and spore properties. J. Bacteriol. 181, 126-132.

30. Meberg, B. M., Paulson, A. L., Priyadarshini, R. \& Young, K. D. (2004). Endopeptidase penicillin-binding proteins 4 and 7 play auxiliary roles in determining uniform morphology of Escherichia coli. J. Bacteriol. 186, 8326-8336.

31. Priyadarshini, R., Popham, D. L. \& Young, K. D. (2006). Daughter cell separation by penicillin-binding proteins and peptidoglycan amidases in Escherichia coli. J. Bacteriol. 188, 5345-5355.

32. Gallant, C. V., Daniels, C., Leung, J. M., Ghosh, A. S., Young, K. D., Kotra, L. P. \& Burrows, L. L. (2005). Common beta-lactamases inhibit bacterial biofilm formation. Mol. Microbiol. 58, 1012-1024.

33. Pedersen, L. B., Murray, T., Popham, D. L. \& Setlow, P. (1998). Characterization of dacC, which encodes a new low-molecular-weight penicillin-binding protein in Bacillus subtilis. J. Bacteriol. 180, 4967-4973.

34. Harris, F., Brandenburg, K., Seydel, U. \& Phoenix, D. (2002). Investigations into the mechanisms used by the C-terminal anchors of Escherichia coli penicillinbinding proteins 4, 5, 6 and $6 \mathrm{~b}$ for membrane interaction. Eur. J. Biochem. 269, 5821-5829.

35. Streshinskaya, G. M., Shashkov, A. S., Usov, A. I., Evtushenko, L. I. \& Naumova, I. B. (2002). Cell wall teichoic acids of actinomycetes of three genera of the order actinomycetales. Biochemistry (Mosc.), 67, 778-785.

36. Jancarik, J., Scott, W. G., Milligan, D. L., Koshland, D. E., Jr \& Kim, S. H. (1991). Crystallization and preliminary $\mathrm{X}$-ray diffraction study of the ligand-binding domain of the bacterial chemotaxis-mediating aspartate receptor of Salmonella typhimurium. J. Mol. Biol. 221, 31-34.

37. McPherson, A. (1999). Crystallization of Biological Macromolecules. Cold Spring Harbor Laboratory Press, Cold Spring Harbor, NY.

38. Leslie, A. G. W. (1991). Molecular data processing. Crystallog. Comput. 5, 50-61.

39. CCP4. (1994). The CCP4 suite: programs for protein crystallography. Acta Crystallog. sect. D, 50, 760-763.

40. Navaza, J. (2001). Implementation of molecular replacement in AMoRe. Acta Crystallog. sect. D, 57, 1367-1372.

41. Brunger, A. T., Adams, P. D., Clore, G. M., DeLano, W. L., Gros, P., Grosse-Kunstleve, R. W. et al. (1998). Crystallography and NMR system: a new software suite for macromolecular structure determination. Acta Crystallog. sect. D, 54, 905-921.

42. Roussel, A. \& Cambillau, C. (1989). Silicon Graphics Geometry Partner Directory. Silicon Graphics, Mountain View, CA.

43. DeLano, W. L. (2002). The PyMOL Molecular Graphics System. DeLano Scientific, San Carlos, CA, USA.

44. Kraulis, P. J. (1991). MOLSCRIPT: a program to produce both detailed and schematic plots of protein structures. J. Appl. Crystallog. 24, 946-950.

45. Esnouf, R. M. (1997). An extensively modified version of MolScript that includes greatly enhanced coloring capabilities. J. Mol. Graph. Model. 15, 132-134, 112-113.

46. Merritt, E. A. \& Murphy, M. E. (1994). Raster3D Version 2.0. A program for photorealistic molecular graphics. Acta Crystallog. sect. D, 50, 869-873.

47. Granier, B., Duez, C., Lepage, S., Englebert, S., Dusart, J., Dideberg, O. et al. (1992). Primary and predicted secondary structures of the Actinomadura R39 extracellular DD-peptidase, a penicillin-binding protein (PBP) related to the Escherichia coli PBP4. Biochem. J. 282, 781-788.

48. Frère, J. M. \& Joris, B. (1985). Penicillin-sensitive enzymes in peptidoglycan biosynthesis. Crit. Rev. Microbiol. 11, 299-396. 